\title{
Active learning accelerates ab initio molecular dynamics on pericyclic reactive energy surfaces
}

\author{
Shi Jun Ang, ${ }^{\dagger}$ Wujie Wang, ${ }^{\dagger}$ Daniel Schwalbe-Koda, ${ }^{\dagger}$ Simon Axelrod, ${ }^{\ddagger}, \dagger$ and \\ Rafael Gómez-Bombarelli*,† \\ $\dagger$ Department of Material Science and Engineering, Massachusetts Institute of Technology, \\ Cambridge, MA 02139, USA \\ $\ddagger$ Department of Chemistry and Chemical Biology, Harvard University, Cambridge, MA \\ 02138, USA \\ E-mail: rafagb@mit.edu
}

\begin{abstract}
Modeling dynamical effects in chemical reactions, such as post-transition state bifurcation, requires $a b$ initio molecular dynamics simulations due to the breakdown of simpler static models like transition state theory. However, these simulations tend to be restricted to lower-accuracy electronic structure methods and scarce sampling because of their high computational cost. Here, we report the use of statistical learning to accelerate reactive molecular dynamics simulations by combining high-throughput ab initio calculations, graph-convolution interatomic potentials and active learning. This pipeline was demonstrated on an ambimodal trispericyclic reaction involving 8,8dicyanoheptafulvene and 6,6-dimethylfulvene. With a training dataset size of approximately 31,000 M062X/def2-SVP quantum mechanical calculations, the computational cost of exploring the reactive potential energy surface was reduced by an order of magnitude. These tools are then used to automatically predict a reaction mechanism that
\end{abstract}


is in agreement with the experimentally-reported product distribution. In addition, thousands of virtually costless picosecond-long reactive trajectories suggest that posttransition state bifurcation plays a very minor role in the reaction. Furthermore, a transfer-learning strategy effectively allowed to upgrade the potential energy surface to higher levels of theory (def2-TZVPD basis set and double hybrid functional) using less than $10 \%$ additional calculations. Since these methods capture intramolecular non-covalent interactions more accurately, they uncover longer lifetimes for entropic intermediates. This overall approach is broadly applicable and opens the door to the study of dynamical effects in larger, previously-intractable reactive systems.

\section{Introduction}

Transition state theory (TST) lies at the heart of most computational chemistry approaches to chemical reactivity. Reaction rates and product selectivities can be quantified, and thus predicted, from the the (free) energies difference between reactants and transition states (TS) whose atomic configurations are essentially taken to be static points on the potential energy surface (PES). Because transition states are first-order saddle points on a high-dimensional PES, locating the lowest TS that connects a reactant and a product with computational methods is a non-trivial task. Typically, approaches need to combine local Hessian-based eigenvector-following (EVF) algorithms ${ }^{1}$ and very strong heuristics with other string-based approaches that require less supervisions, such as nudged elastic band (NEB), ${ }^{2}$ freezing ${ }^{3,4}$ and growing ${ }^{5}$ string methods. The static nature of TST, however, fails to explain dynamical effects that are responsible for the product distribution of certain synthetic and biosynthetic reactions. ${ }^{6}$ The computational study of these dynamical effects requires ab initio molecular dynamics (AIMD). ${ }^{7,8}$ In this approach, nuclei are treated as Newtonian bodies and a coupled

electronic structure problem is solved with density functional theory (DFT) or wavefunction methods to find the PES. AIMD simulations using Gaussian bases are typically limited to Born-Oppenheimer MD (BOMD), ${ }^{9,10}$ whereas in plane wave bases some acceleration is pos- 
sible with the Car-Parrinello (CP) method. ${ }^{11}$ Path-integral molecular dynamics can capture quantum nuclear effects in challenging systems. ${ }^{12-14}$

Many pericyclic reactions ${ }^{15,16}$ have been chosen as examples to illustrate the post-TS dynamical effects due to the possibility of multiple addition/rearrangement end products, and the relatively abundant experimental data on a variety of substrates and mechanisms. In this case, the selectivity of one product over another is called periselectivity. Recently, Singleton, ${ }^{17}$ Tantillo, ${ }^{18}$ Paton ${ }^{19}$ and Houk ${ }^{20}$ utilized BOMD extensively to study pericyclic reactions that show post-TS bifurcations. These are reactions in which a single TS leads directly to two (or more) products because of a bifurcating, relatively flat PES after the relevant TS. ${ }^{20}$ The product selectivity of such reactions cannot be explained with traditional TST, as they favor the formation of entropic intermediates with sub-picosecond lifetimes. These intermediates are not local energy minima but minima on the corresponding Gibbs free energy surfaces. ${ }^{20}$ In general, BOMD simulations are computationally expensive due to the combination of (i) very small integration time steps (femtosecond or lower) compared with the timescale of chemical reactions, (ii) the inherent cost, and scaling, of the electronic structure methods used to calculate energies and forces, (iii) the need to model an ensemble of reactive trajectories. BOMD simulations are typically limited to systems with a few dozen atoms, for which tens of trajectories totalling tens to hundreds of picoseconds are acquired with hybrid exchange-correlation functionals and small double-zeta basis sets, needing in the order of $10^{4}-10^{5}$ SCF energy and gradient calls.

The recent advances in machine learning (ML) techniques and the modularization of neural network $(\mathrm{NN})$ routines $^{21,22}$ facilitate the interplay between physical sciences and statistical learning. These techniques provide opportunities to speed up the costly BOMD simulations by several orders of magnitudes. NN-based interatomic potentials built on a variety of molecular representations retain the accuracy of the $a b$ initio training data and can be executed at computational cost similar to classical force fields. To date, a variety of NN-based force fields have been developed, starting from the seminal Behler-Parrinello 
approach, ${ }^{23}$ ANI, ${ }^{24-27}$ Deep Tensor Neural Network, ${ }^{28}$ SchNet $^{29,30}$ Deep Potential Net, ${ }^{31}$ and MEGNet. ${ }^{32}$ These potentials can effectively learn short-range many-body interactions in all-atom and coarse-grained potentials. ${ }^{33,34}$ In their recent review, Noé and Jensen covered important aspects of accelerating molecular simulations using ML techniques. ${ }^{35,36}$ Particularly in the area of surface catalysis and solid-state diffusion, ML approaches have been proposed to accelerate the location of transition states and exploration of minimum energy paths. ${ }^{37-39}$ Similarly, high-throughput simulation approaches that have, for organics, been typically aimed at static properties of single molecules, ${ }^{40-43}$ are increasingly targeting reactivity. ${ }^{44-48}$

Here, we demonstrate the combined application of high-throughput (HT) simulation and bootstrapped ML interatomic potentials to study a complicated reactive system that is dominated by dynamical effects. Specifically, we studied the pericyclic reactions involving 8,8-dicyanoheptafulvene and 6,6-dimethylfulvene. Experimental results ${ }^{49}$ at room temperature in chloroform for one day, afford a product distribution, of $0: 11.5: 9.8$ for Product $1[4+6]$ : Product $2 \mathrm{a}[6+4](+$ tautomer) : Product $2 \mathrm{~b}[8+2]$. Recently, Xue et al. carried out TS and BOMD simulations of the same reaction in the gas phase. ${ }^{50}$ Figure 1 shows the major product of each of the reactions. For reaction 1, 87\% of the 142 trajectories resulted in Product 1, and 3\% each for Products $2 \mathrm{a}$ and $2 \mathrm{~b}$. For reactions $2 \mathrm{a}$ and $2 \mathrm{~b}$, the majority of the trajectories were reported to result in products $2 \mathrm{a}$ and $2 \mathrm{~b}$ respectively.

The key components of the proposed approach are coupled loops of (i) high-throughput (HT) simulation for rapid, parallel acquisition of DFT data; (ii) the training of ML interatomic potentials based on graph convolutions neural networks ${ }^{29,51,52}$ (iii) and the rapid inference to sample configurational space with NN-based versions of EVF, NEB, and reactive MD. The converged interatomic potential is trained on a total of 30,973 M062X/def2-SVP configurations and recovers the reaction PES with near $\mathrm{kJ} \mathrm{mol}^{-1}$ accuracy. To investigate the role of the level of theory, we leveraged a transfer-learning strategy ${ }^{25}$ to a larger-basis set (M06-2X/def2-TZVPD) and double-hybrid functional (DLPNO-DSD-PBEP86-D3BJ/def2- 
TZVPD, abbreviated as DH/def2-TZVPD) using less than $10 \%$ additional training points for each. Both transfer-learned potentials attained out-of-sample sub-kcal mol ${ }^{-1}$ accuracy.

Through thousands of picosecond NRMD trajectories at the varying levels of theory, we conclude that the role of bifurcation is very minor in explaining the product distribution of the pericyclic reaction of 8,8-dicyanoheptafulvene and 6,6-dimethylfulvene. The use of the larger def2-TZVPD basis set, which includes diffuse functions, and the double-hybrid functional captures intramolecular non-covalent interactions more accurately ${ }^{53}$ and results in longer-lived entropic intermediates than the smaller basis. Lastly, the role of the solvent bath is explored by performing Langevin dynamics at two friction coefficients (0.0012 a.u. and 0.024 a.u.). It is observed that bifurcation only becomes significant at unrealistically large friction coefficients, which also result in increased lifetimes of the entropic intermediate.

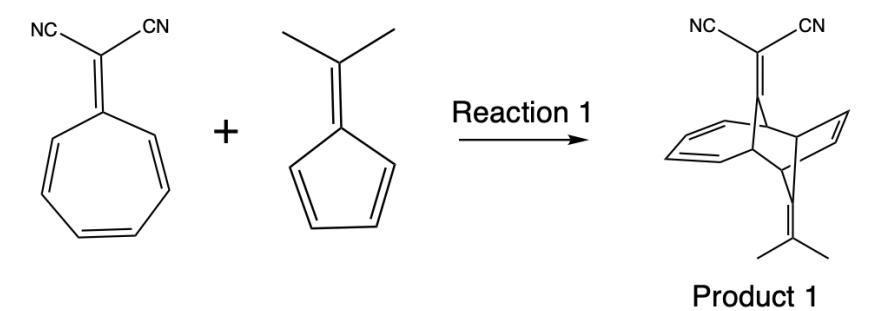

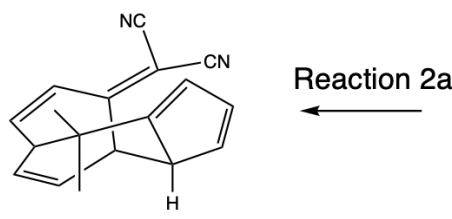

Product 2a

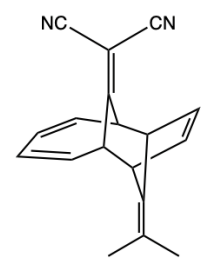

Product 1

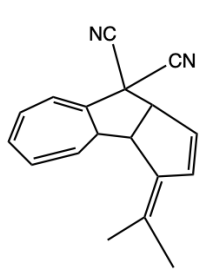

Product $2 b$

Figure 1: Three distinct reactions studied in this manuscript

\section{Methodology}

\section{Quantum Chemical Calculations}

All quantum chemical calculations were carried out using ORCA 4.1.1. ${ }^{54,55}$ M06-2X/def2SVP was chosen as the default level of theory for all calculations. For transfer learning, M06- 
2X/def2-TZVPD and the higher rung double-hybrid DLPNO-DSD-PBEP86 D3BJ/def2TZVPD were used. M06-2X is a standard, ${ }^{56}$ well-benchmarked choice, ${ }^{53}$ due to its accuracy in predicting reaction barriers. As the Minnesota functionals converge slowly with DFT integration grids, we used a very fine option (Grid6) in all computations. ${ }^{57,58}$ All NEB computations were carried out with 16 intermediate (moving) images. The starting paths were generated with the image-dependant pair potential (IDPP) algorithm, which interpolates the

corresponding pairwise interatomic distances between the defined reactant and product. ${ }^{59}$ The default spring constant of $0.1 \mathrm{Ha} \mathrm{Bohr}^{-2}$ and the improved tangent method for the calculation of parallel forces were employed. The L-BFGS algorithm was used for optimization. Harmonic analysis was performed to ensure that geometries show the expected number of negative eigenvalues.

\section{Initial guesses for Quantum Chemical NEB}

Individual molecules were entered into an PostgreSQL database via SMILES strings. These SMILES were converted into 3D structures using RDKit. ${ }^{60}$ The respective reactant(s) and product pairs were then linked using SMARTS. Corresponding atoms in the reactant(s) and product were mapped using the SMARTS map classes, since correct ordering of atoms is essential for generating the correct root-mean-squared deviation between structures (RMSD) and to run NEB calculations. For reaction 1 involving two reactants and one product, 50,000 random reactive dimers were generated using the MMFF94-optimized ${ }^{61}$ structures of the reactants. RMSD values were then calculated between dimer structures and the structure of the product optimized with the same method. The reactive dimer with the lowest RMSD with respect to the product was chosen as the starting point for the IDPP interpolation before Quantum Mechanical NEB.

For reactions $\mathbf{2} \mathbf{a}$ and $\mathbf{2} \mathbf{b}$, there are one reactant and one corresponding product respectively. 5,000 unoptimized conformers were generated for each molecule and the reactantproduct pairs with the lowest RMSD were chosen. 


\section{Active learning}

\section{Outline}

After obtaining the aforementioned reactive complexes and index-matched product geometries, 3 independent active learning loops were constructed, one per reaction. For each round of neural reactive $\mathrm{MD}$, trajectories were propagated with the learned interatomic potential starting from the propagating images of the final neural NEB band (highest image $\pm n$, where $n=2,3,4$ or 5 ). The exact value of $n$ was determined by a trial MD run to ensure that the MD trajectories proceeded towards the reactant and product for the forward and backward propagating trajectories respectively. The first rounds of NRMD were carried out for 100 fs in each direction with stepsize of 0.1 fs starting from quantum chemical NEB images and using an interatomic potential trained with the results from the quantum chemical NEB augmented with 1,600 displaced geometries along the NEB string. MD frames were saved every 10 steps. Subsequent rounds were carried out for 500 fs in each direction with stepsize of 1 fs, starting from neural NEB simulations with the prior-generation interatomic potential. Frames were saved every 5 fs. 5 pairs of MD trajectories were propagated after each neural NEB run. Energies and atomic forces for the selected frames were then calculated with DFT and added to the pool of training data for each reaction. The active learning loops were terminated after 5 training iterations. A final model was trained on all acquired datapoints from the three independent active learning loops. All models were trained by partitioning data into training (60\%), validation (20\%) and test sets (20\%). Structures generated from NRMDs with gradient element(s) of absolute values greater than $0.15 \mathrm{Ha} \mathrm{bohr}^{-1}$ were discarded. The final number of datapoints was 30,973. As a comparison, the total number of energy gradient calculations performed by Xue et al. during the BOMD simulations is about 245,000, eight times larger than ours. Furthermore, DFT simulations in each generation of the active learning loop are uncorrelated and embarrasingly parallel. Hence, they are more resource-efficient than $\mathrm{BOMD}$, which forces to sample the PES in series and relies on the 
parallelization of DFT codes. A depiction of an active learning loop is shown in Figure 2.

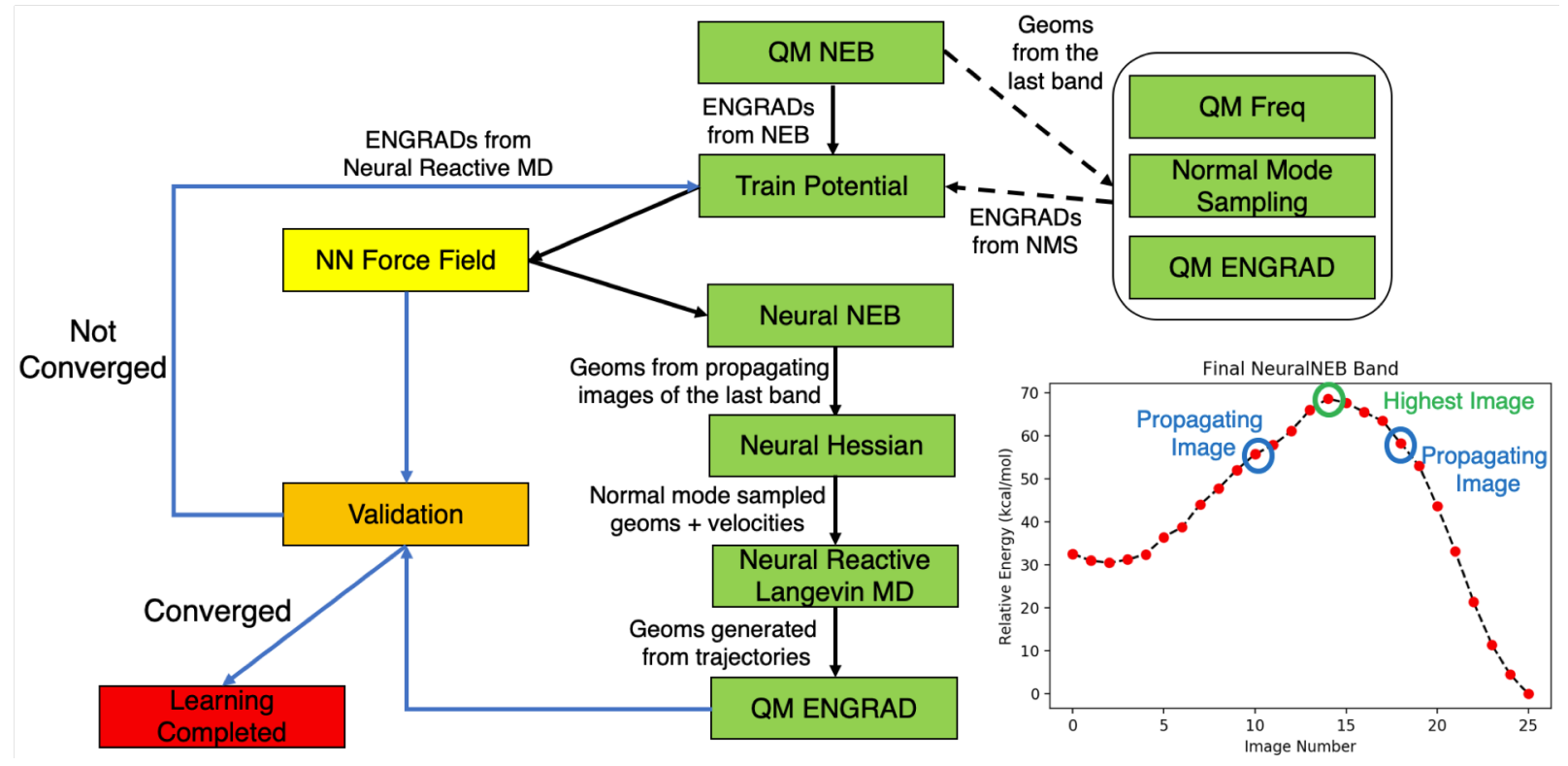

Figure 2: Overall outline of the active learning procedure. Data acquisition starts from quantum chemical NEB for a particular reaction, by storing molecular energies and atomic forces throughout the NEB cycles. Upon completion, each image from the final NEB band was displaced with normal mode sampling (100 displaced geometries for each image). DFT energies and forces were acquired for these geometries. The NEB and the normal mode sampled data points were then combined for the first round NN training. After which, the active learning loop self-sustains using neural NEB, neural hessian and NRMD sequentially with trained NN at every generation. This pipeline generates more geometries in which DFT energies and forces were collected. In the following loop, the newly generated datapoints were added to previous datapoints for the training of an updated NN.

\section{Neural Network Architecture}

We adopted the SchNet ${ }^{29,30}$ architecture, a model based on graph convolutions, to generate interatomic potentials. The model consists of a convolution component and a readout component. The convolution component creates an atomic fingerprint for each atom in the molecule, based on its atomic number and its position relative to other atoms. The readout section consists of two fully connected layers that act on each atomic fingerprint to produce an energy for each atom. The total energy is the sum of each atomic energy.

The atomic fingerprint of each atom is initialized with a vector embedding of its atomic 
number. Each atomic number has a unique embedding vector, which is initially a set of random numbers. Note that the embedding vector is updated in the process of training, so that features shared by certain atomic numbers become represented in the embeddings. After embedding, the atomic fingerprint is updated to account for the influence of its environment. This so-called convolution is performed as follows. First, a filter network is generated. This network is a representation of the local environment. A pre-defined cutoff radius is set, and the interatomic distance between each pair of atoms in the region is expanded in a basis of Gaussian functions. Next, a set of non-linear (shifted-softplus) operations is applied to the Gaussian functions to create a set of filter vectors for each pair of atoms. Finally, each filter vector coupling an atom to its neighbors is multiplied by the neighbor's fingerprint and added to the atom's fingerprint. A set of non-linear functions is applied to the fingerprint before and after this operation.

A series of convolutions is performed to update the original embedding vector. The first convolution accounts for interactions of each atom with other atoms within the cutoff radius. The second convolution accounts for interactions within twice the cutoff radius, the third with three times the cutoff radius, and so on. After the final atomic fingerprints are generated, a set of two fully connected layers (i.e., a multi-layer perceptron, or MLP) is applied to each fingerprint. This generates a set of atomwise energies, which are added to give the total energy. The SchNet architecture is invariant to translation, rotation, and permutation of atomic indices. The former two are guaranteed by the fact that only interatomic distances are used to produce the energy. The latter is ensured by the fact that summation of atomic energies is invariant to atom relabeling.

Here we use five convolutions, a cutoff radius of $5.5 \AA$, a dimension of 256 for the atomic feature vector, and 32 Gaussian functions for the distance expansion. Tunable parameters were optimized using the Adam algorithm ${ }^{62}$ with batch size of 32 , initial learning rate of $3 \times 10^{-4}$, learning rate decay of 0.5 and learning rate patience of 25 . Training was terminated when the learning rate reached $2 \times 10^{-7}$. 
DFT forces were used without modifications. For energies, a thermodynamic reference state was chosen to limit the absolute range of energy values. The reference energies of $\mathrm{C}$, $\mathrm{N}$ and $\mathrm{H}$ were evaluated using the M06-2X/def2-SVP energies of $\mathrm{CH}_{4}, \mathrm{~N}_{2}$ and $\mathrm{H}_{2}$.

\section{Neural NEB}

NN interatomic potentials were interfaced with the NEB module available in the Atomic Simulation Environment (ASE) package. ${ }^{63}$ All neural NEB calculations were performed with 24 intermediate images (not including reactant and product structures, which are kept fixed throughout the NEB process). The convergence criterion was a maximum force below 4 $m e V \AA^{-1}$. The BFGS algorithm was used for Hessian update during the optimization process. The spring force was set to $50 \mathrm{meV} \AA^{-2}$. Parallel forces were computed using the improved tangent method. ${ }^{64}$

\section{Neural Eigenvector Following}

The projected rational function optimization method of Baker ${ }^{1}$ was implemented in PyTorch to perform neural eigenvector following (EVF) simulations. In the first iteration, the numerical Hessian was calculated using finite-difference with the vibrations package in ASE. For subsequent iterations, the Powell Update was used to estimate the Hessian.

\section{Neural Reactive MD}

Trajectories were propagated forward and backward from normal mode sampled geometries of the various QM transition states ${ }^{65}$ for $500 \mathrm{fs}$ in each direction at $\mathrm{T}=298.15 \mathrm{~K}$ with a timestep of $1 \mathrm{fs}$. 1,000 pairs of trajectories were propagated from each of the three transition states. The Langevin thermostat (NVT) was used to mimic mechanical effects of solvent molecules. The Langevin friction coefficient was estimated using the experimental diffusion coefficient of benzene in chloroform, $2.89 \mathrm{~cm}^{2} \mathrm{~s}^{-1} .{ }^{66}$ The associated friction coefficient $(\gamma=$ 0.0012 a.u.) was calculated using Equation 1, where $\gamma$ is the friction coefficient, $k_{B}$ is the 
Boltzmann constant, and $D$ is the diffusion coefficient.

$$
\gamma=\frac{k_{B} T}{D}
$$

In order to study the effect of Langevin friction coefficient on the kinetic product distribution and lifetime of the entropic intermediate, we performed an additional set of NRMD simulations each with the transfer-learned NNs with $\gamma$ values of 0.024 a.u. (20 times larger).

\section{Transfer Learning}

Two sets of 3,093 and 3,067 structures were selected at random from the final dataset obtained using M06-2X/def2-SVP level of theory via previous rounds of active learning loops. DFT energy and forces calculations were performed at M06-2X/def2-TZVPD and a more accurate double-hybrid DLPNO-DSD-PBEP86-D3BJ/def2-TZVPD for the respective sets of structures. The weights optimized for the smaller basis set in the convolutional and interaction layers were frozen. The weights from two fully-connected readout layers were randomly initialized and optimized with the datapoints calculated using the larger basis set. The available dataset was randomly split into training (60\%), validation (20\%) and test (20\%). Hyperparameters were held constant.

\section{Results and discussion}

\section{Active Learning}

The summary of the three independent sets of active learning cycles is presented in Table 2. The mean absolute errors (MAEs) of the test set provide a measure of the performance of the trained $\mathrm{NN}$ with respect to the datapoints similar to those used for training. On the other hand, the trained NNs at each active learning cycle were used to acquire additional datapoints through NRMD, starting from the propagating images of a neural NEB performed with the 
same NN. Thus, the MAEs of the NRMD datapoints can be seen as a more appropriate "test set" that is dissimilar to the held out test set. At the end of cycle 5 of the active learning loops, all energy and energy gradient MAEs fell below $1.0 \mathrm{kcal} \mathrm{mol}{ }^{-1} / \mathrm{kcal} \mathrm{mol}^{-1} \AA^{-1}$ and slightly higher for non-holdout data.

Table 1: Mean absolute errors (MAE) for individual active learning loop

\begin{tabular}{|c|c|c|c|c|c|c|}
\hline \multicolumn{7}{|c|}{ Energy (kcal mol $\left.{ }^{-1}\right)$} \\
\hline \multirow{2}{*}{$\begin{array}{c}\text { Active Learning } \\
\text { Cycle }\end{array}$} & \multicolumn{2}{|c|}{ Reaction 1} & \multicolumn{2}{|c|}{ Reaction $2 \mathrm{a}$} & \multicolumn{2}{|c|}{ Reaction $2 \mathrm{~b}$} \\
\hline & Test & NRMD & Test & NRMD & Test & NRMD \\
\hline 1 & 1.00 & 30.66 & 0.42 & 33.74 & 0.23 & 55.69 \\
\hline 2 & 0.35 & 29.91 & 0.17 & 5.89 & 0.15 & 13.29 \\
\hline 3 & 0.40 & 2.30 & 0.84 & 1.53 & 0.21 & 7.90 \\
\hline 4 & 0.53 & 1.25 & 0.18 & 0.80 & 0.21 & 1.74 \\
\hline 5 & 0.55 & 1.18 & 0.16 & 0.65 & 0.27 & 0.68 \\
\hline \multicolumn{7}{|c|}{ Energy Gradient (kcal mol $\left.{ }^{-1} \AA^{-1}\right)$} \\
\hline \multirow{2}{*}{$\begin{array}{c}\text { Active Learning } \\
\text { Cycle }\end{array}$} & \multicolumn{2}{|c|}{ Reaction 1} & \multicolumn{2}{|c|}{ Reaction $2 \mathrm{a}$} & \multicolumn{2}{|c|}{ Reaction 2b } \\
\hline & Test & NRMD & Test & NRMD & Test & NRMD \\
\hline 1 & 0.56 & 7.06 & 0.26 & 5.01 & 0.57 & 8.61 \\
\hline 2 & 0.64 & 9.59 & 0.26 & 3.14 & 0.20 & 3.52 \\
\hline 3 & 0.61 & 2.34 & 0.47 & 2.06 & 0.44 & 2.63 \\
\hline 4 & 0.72 & 1.91 & 0.38 & 1.54 & 0.54 & 2.43 \\
\hline 5 & 0.51 & 1.58 & 0.45 & 1.41 & 0.54 & 1.15 \\
\hline Total datapoints generated & \multicolumn{2}{|c|}{7393} & \multicolumn{2}{|c|}{10987} & \multicolumn{2}{|c|}{12593} \\
\hline
\end{tabular}

\section{Consolidated Model}

The final training utilizing 30,973 datapoints resulted in excellent agreement with the reference M06-2X/def2-SVP energies and forces (Figure 3). For the held out test set, the MAEs for both energy $\left(0.173 \mathrm{kcal} \mathrm{mol}^{-1}\right)$ and energy gradient $\left(0.236 \mathrm{kcal} \mathrm{mol}^{-1} \AA^{-1}\right)$ exceeded sub-chemical accuracy of $0.24 \mathrm{kcal} \mathrm{mol}^{-1}$ and $\mathrm{kcal} \mathrm{mol}^{-1} \AA^{-1}$ respectively. This indicates that the NN architecture is sufficiently flexible to learn the complex reactive PES of this pericyclic reaction. This optimized neural network is abbreviated as NN-small, denoting the small def2-SVP basis set used for the DFT data. 

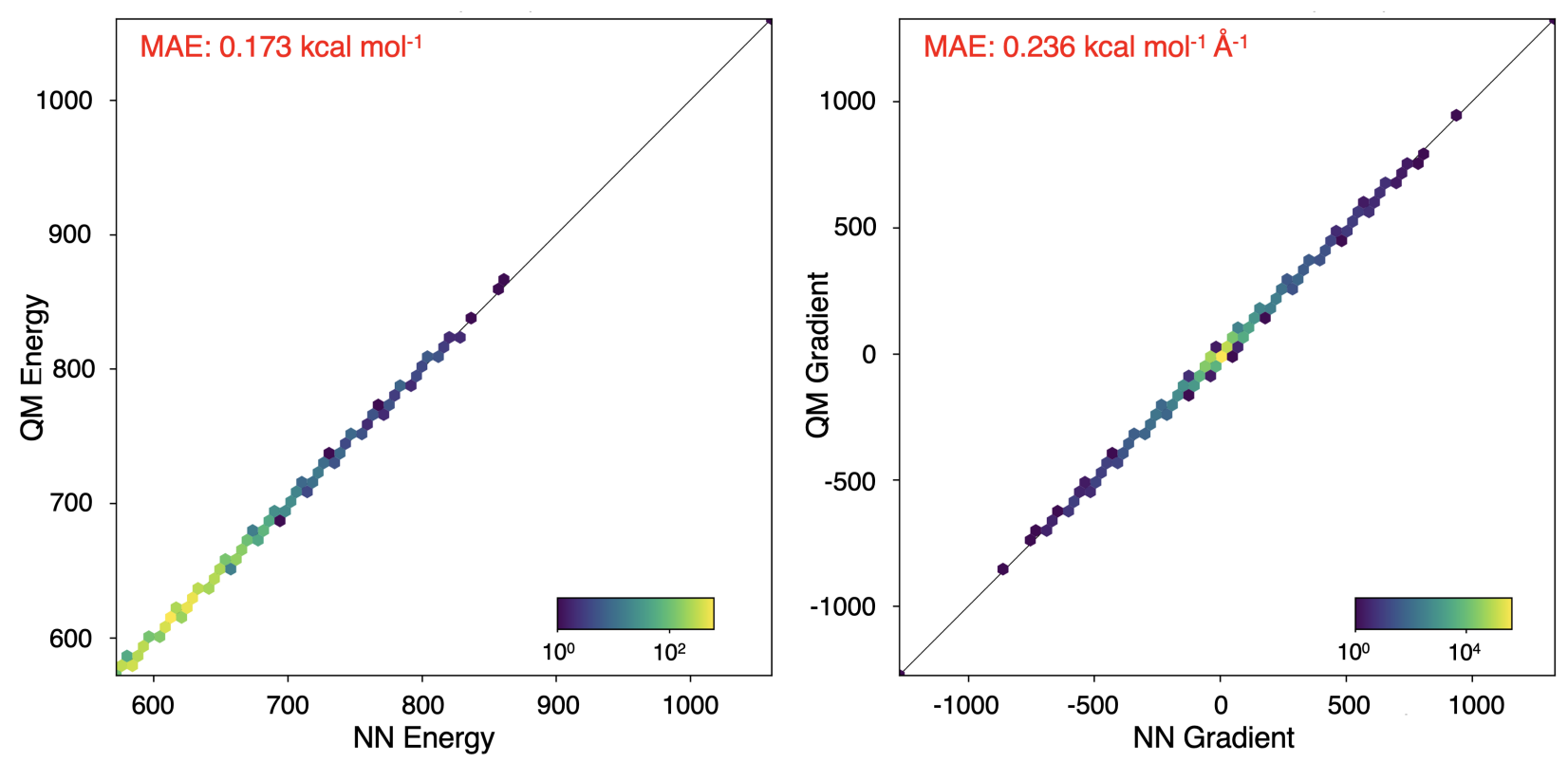

Figure 3: Mean absolute error for energy $\left(\mathrm{kcal} \mathrm{mol}^{-1}\right)$ and energy gradient(kcal mol $\left.{ }^{-1} \AA^{-1}\right)$

\section{Transition State Structures and Energetics}

The highest-energy image in each of the three converged bands arising from the last-generation neural NEBs (Figure S2) were chosen as transition-state guess geomtries and further refined neural EVF followed by DFT TS optimizations. Comparing the starting and the final structures of the neural EVF optimizations, the RMSD values with respect to the QM structures were reduced by 0.2 to $0.7 \AA$ (Figure S3). This suggest that the PES is well captured by the NN, and also that NEB algorithm, even with a large number of images, produces only approximate guesses for the TS structure.

The important bond lengths specified in the Figure 4 agree well reported $\omega$ B97X-D/6$31 \mathrm{G}(\mathrm{d})$ structures. $^{50} \mathbf{T S} \mathbf{2 a}$ and $\mathbf{T S} \mathbf{2 b}$ are found to be 2.3 and $1.4 \mathrm{kcal} \mathrm{mol}^{-1}$ lower in energy that TS 1 respectively, in keeping with previously reported $\omega$ B97X-D/6-31G(d) values of 2.7 and $1.7 \mathrm{kcal} \mathrm{mol}^{-1}$. Furthermore, the NN and QM relative energy and several other thermochemical quantities at various QM stationary points showed good agreement (Table 2), with a maximum error in electronic energies of $0.8 \mathrm{kcal} \mathrm{mol}^{-1}$. Both NN zero-point energies and enthalpy corrections also achieved sub-kcal mol ${ }^{-1}$ accuracy. Underestimation 
of the vibrational frequency of low-energy vibrations in the harmonic approximations results in larger errors in NN entropic corrections. The quasi-harmonic entropy corrections of Truhlar ${ }^{67}$ or Grimme ${ }^{68}$ could be applied to produce better estimates. Individual hessian matrix elements were validated and presented in Figure S4. The NN values were in very good agreement with the corresponding QM ones (RMSEs are $0.15 \mathrm{kcal} \mathrm{mol}^{-1} \AA^{-2}$ for all transition states and $0.16 \mathrm{kcal} \mathrm{mol}^{-1} \AA^{-2}$ for all minima).

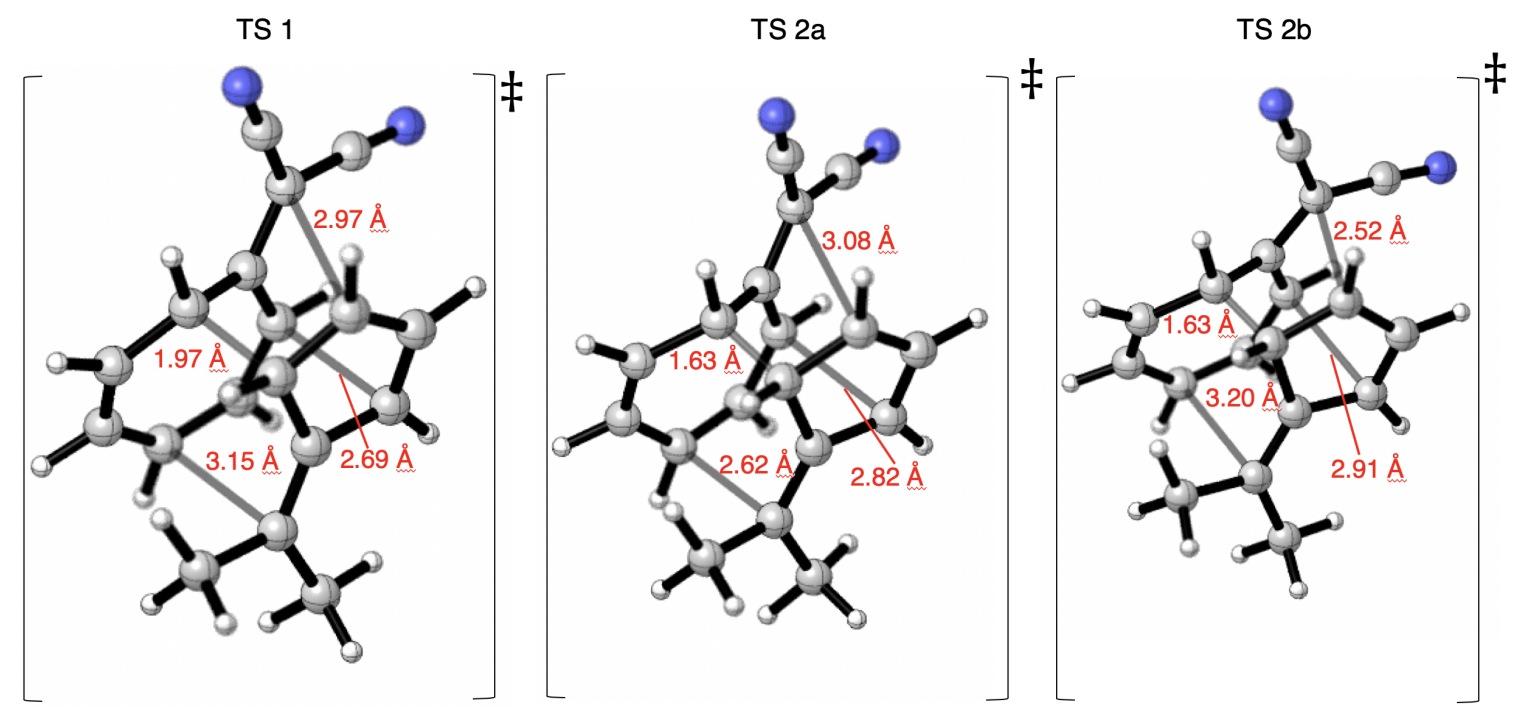

Figure 4: Important bond lengths of M06-2X/def2-SVP optimized TS structures

Table 2: NN relative energy, zero-point energy, enthalpy correction (without ZPE), entropy correction and Gibbs free energy correction $\left(\mathrm{kcal} \mathrm{mol}^{-1}\right), \mathrm{T}=298.15 . \mathrm{G}_{\mathrm{corr}}=\mathrm{ZPE}+\mathrm{H}_{\text {corr }}$ - $\mathrm{TS}_{\text {corr }}$. Corresponding QM values in parentheses.

\begin{tabular}{lccccc}
\hline Species & Relative Energy & ZPE & $\mathrm{H}_{\text {corr }}$ & $\mathrm{TS}_{\text {corr }}$ & $\mathrm{G}_{\text {corr }}$ \\
\hline Reactants & $0.0(0.0)$ & $180.8(181.1)$ & $12.7(12.5)$ & $56.5(55.0)$ & $136.9(138.7)$ \\
TS 1 & $8.4(7.6)$ & $181.1(181.9)$ & $12.2(11.9)$ & $43.9(39.1)$ & $149.6(154.7)$ \\
Product 1 & $-19.5(-19.6)$ & $184.4(184.4)$ & $11.6(11.6)$ & $39.5(38.5)$ & $156.5(157.4)$ \\
TS 2a & $5.6(5.4)$ & $182.3(182.6)$ & $11.5(11.4)$ & $39.3(37.9)$ & $154.4(156.0)$ \\
Product 2a & $-27.2(-27.4)$ & $184.3(185.1)$ & $11.3(11.1)$ & $38.7(37.2)$ & $156.9(159.0)$ \\
TS 2b & $6.1(6.2)$ & $182.9(182.6)$ & $11.3(11.5)$ & $38.0(38.3)$ & $156.2(155.8)$ \\
Product 2b & $-23.4(-24.1)$ & $184.4(183.7)$ & $11.8(11.7)$ & $40.9(39.0)$ & $155.2(156.5)$ \\
\hline
\end{tabular}




\section{Neural Reactive MD for Ambimodal Trispericyclic Cycloaddition}

The backward and forward Langevin NRMD trajectories starting from the vicinity of a TS can be concatenated (with time reversal) into a complete continuous trajectory that, typically, connects two distinct minima. In addition, trajectories corresponding to "recrossing" and "hovering" were also observed. The "recrossing" trajectories from a PES minimum and connect back to the same minimum after reaching the TS region. This effect has been described extensively by the BOMD community, and cannot be accounted for by TST. In our study, there are also a a small number of trajectories that begin from a particular minimum and hover around regions of the PES corresponding to the entropic intermediate without forming the second bond before the end of the NRMD simulation. There are rarely seen in ab initio MD simulations of organic reactions, and are likely due to the inclusion of the friction term under the Langevin NVT framework.

We number some important atoms of the 36-atom reactive complex/molecule as presented in Figure 5. For all products, a $\sigma$-bond between C1-C2 was always formed, whereas C3-C4, C5-C6 and $\mathrm{C} 7-\mathrm{C} 8 \sigma$-bonds were formed for products $\mathbf{1}, \mathbf{2} \mathbf{a}$ and $\mathbf{2 b}$ respectively.

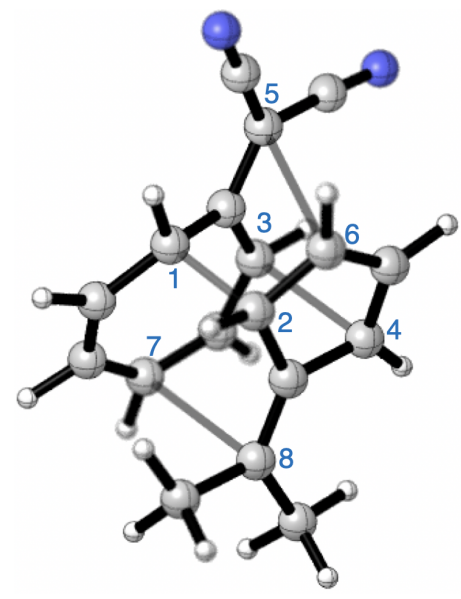

Figure 5: Numbering of important carbon atoms 


\section{Trajectories propagated from the vicinity of TS1}

Out of 1000 trajectories that were initiated from the vicinity of TS 1. Of these, 909 tra-

jectories connected the reactants with product $\mathbf{1}$, and a single one recrossed back to the reactants (Figure 6a). The rest of the 90 trajectories are not chemically relevant as they do not have the reactants as one of their endpoints.

This is in contrast with the BOMD study by Xue et al., where a product ratio of $87: 3$ : 3 for 1 : 2a : $\mathbf{2 b}$ was reported, while the remaining $7 \%$ of the trajectories recrossed back to the reactants. This can be attributed to the difference in level of theory and the use of an MD thermostat.

Figure $6 \mathrm{~b}$ shows a violin plot of time gap between formations of $\mathrm{C} 1-\mathrm{C} 2$ and $\mathrm{C} 3-\mathrm{C} 4$. The time gap is defined as the difference between the times when the bonds of interest first fall below $1.7 \AA$. Out of the 909 trajectories that ended up as product 1, 200 (22\%) trajectories are dynamically concerted ( $<60$ fs between bond formations), the rest are dynamically stepwise. The mean, standard deviation and median of time gaps between the bond formations are 82, 32 and 77 fs respectively. The mean time gap (between the formation of $\mathrm{C} 1-\mathrm{C} 2$ and C3-C4 bonds) of our NRMD simulations are of the same magnitude than the corresponding reported BOMD timegaps (90 fs).

\section{Trajectories propagated from the vicinity of TS $2 \mathrm{a}$ and $2 \mathrm{~b}$}

Since products $\mathbf{2 a}$ and $\mathbf{2} \mathbf{b}$ are formed from product $\mathbf{1}$ via $\mathbf{T S} \mathbf{2 a}$ and $\mathbf{T S} \mathbf{2} \mathbf{b}$, a trajectory was considered valid if it had product 1 on one of its ends. 947 and 1000 valid trajectories originated from product $\mathbf{1}$ via TS $\mathbf{2 a}$ and $\mathbf{T S} \mathbf{2 b}$ respectively (Figures $6 \mathrm{c}$ and d). For the valid trajectories originating from TS 2a, 906 (95.7\%) afforded product 2a, while 37 (3.9\%) recrossed back to product 1. 4 trajectories did not reach a minimum and were classified as hovering. On the other hand, valid trajectories originating from product $\mathbf{1}$ via $\mathbf{T S} \mathbf{2 b}$ have $643(64 \%)$ of them recrossed back to product 1 and only 351 (35.1\%) proceeded to product $\mathbf{2 b}$. This is because the neural PES near $\mathbf{T S} \mathbf{2} \mathbf{b}$ is flatter than that of $\mathbf{T S} \mathbf{2} \mathbf{a}$, as 
the magnitude of the neural imaginary frequency is smaller for the former $\left(136 i \mathrm{~cm}^{-1}\right)$ as compared the latter $\left(197 i \mathrm{~cm}^{-1}\right)$. The corresponding imaginary frequencies at M06-2X/def2SVP level of theory are $168 i$ and $190 i \mathrm{~cm}^{-1}$. These results agree qualitatively with the slightly higher experimental yield of product $\mathbf{2 a}$ as compared to $\mathbf{2} \mathbf{b}$.

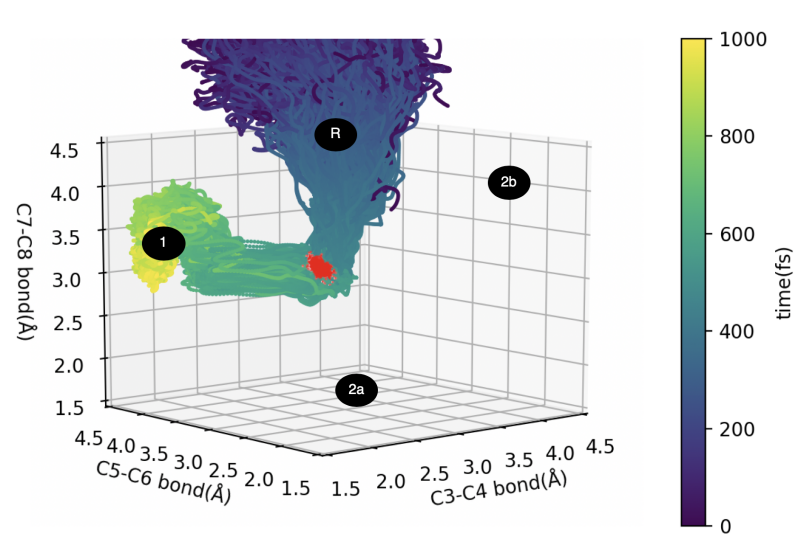

(a) TS 1

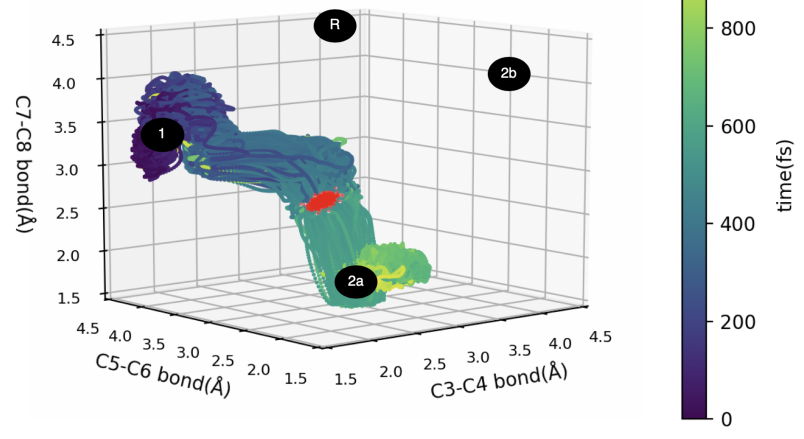

(c) TS $2 \mathrm{a}$

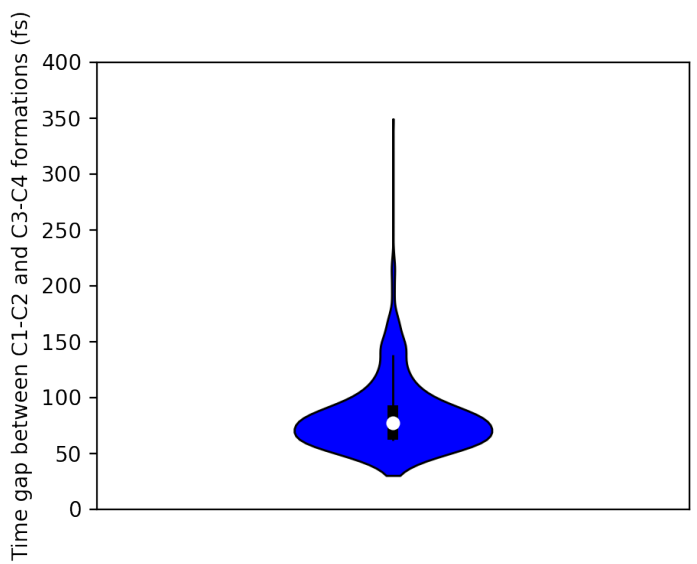

(b) Violin plot of time gap

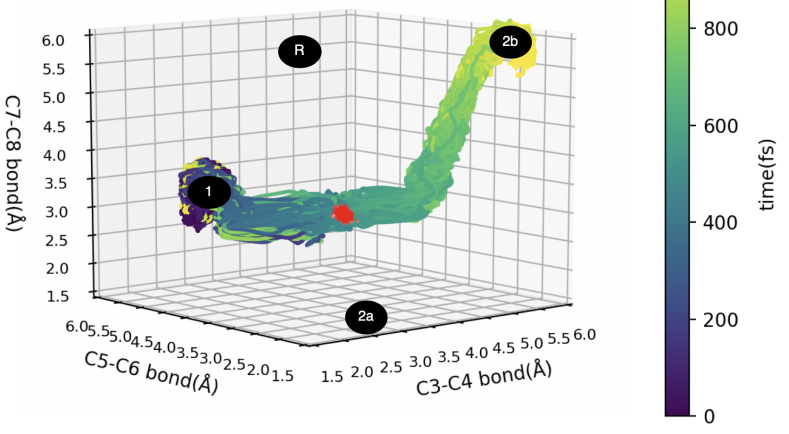

(d) TS $2 b$

Figure 6: Trajectories initiated from the vicinity of TS 1 (a), TS 2a (c), TS 2b (d) using NN-small. Violin plot (b) showing the time gap betweeen C1-C2 and C3-C4 bond formations obtained from trajectories depicted in (a)

\section{Transfer Learning to high level of theory}

\section{Training results}

Figure 7a shows the test statistics of the transfer-learned NN potential using DFT energies and forces acquired with M06-2X/def2-TZVPD. The transfered model performs well, with 
both MAEs of energy $\left(0.537 \mathrm{kcal} \mathrm{mol}^{-1}\right)$ and energy gradient $\left(0.665 \mathrm{kcal} \mathrm{mol}^{-1} \AA^{-1}\right)$ better than "chemical accuracy". We name the transfer-learned neural network NN-large to highlight the larger def2-TZVPD basis set used for training.
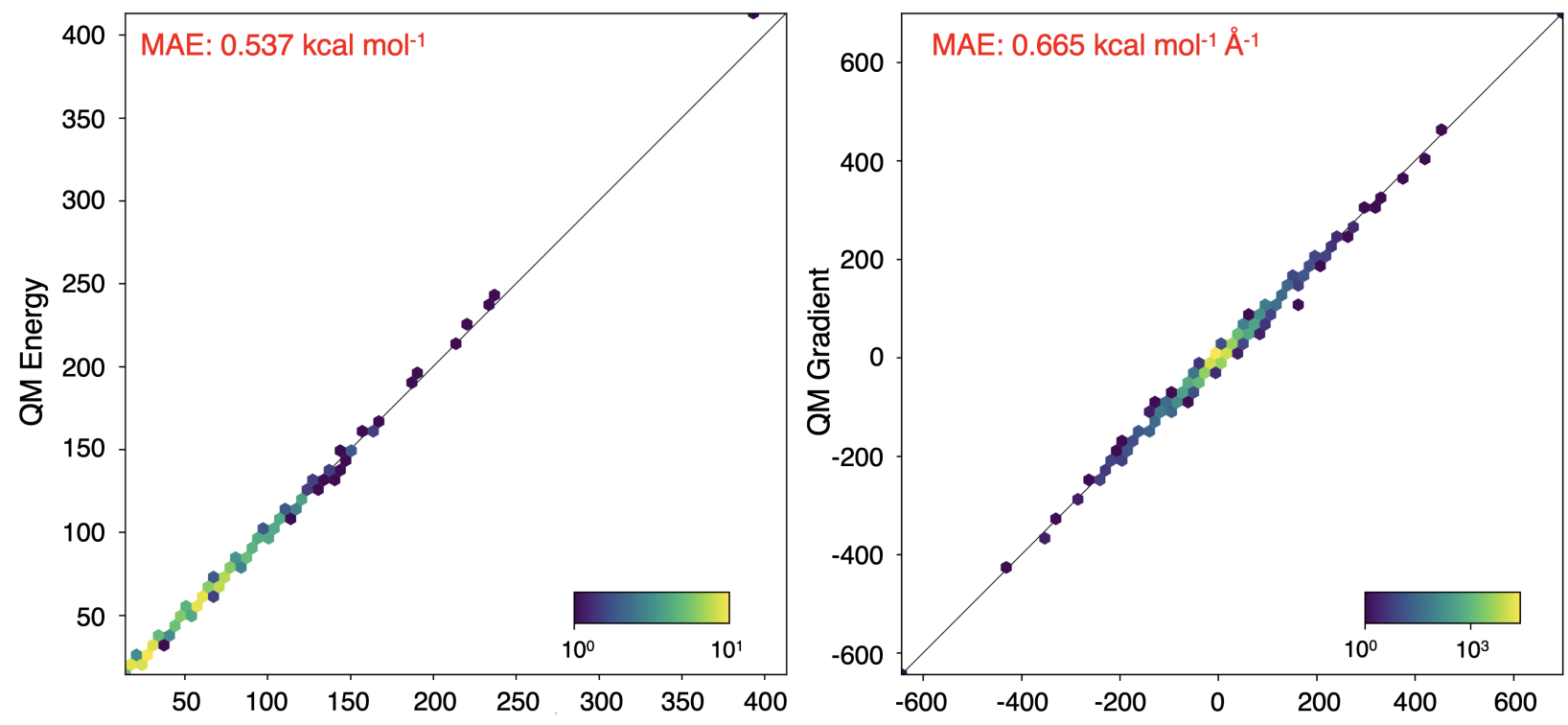

(a) M06-2X/def2-TZVPD (NN-large)
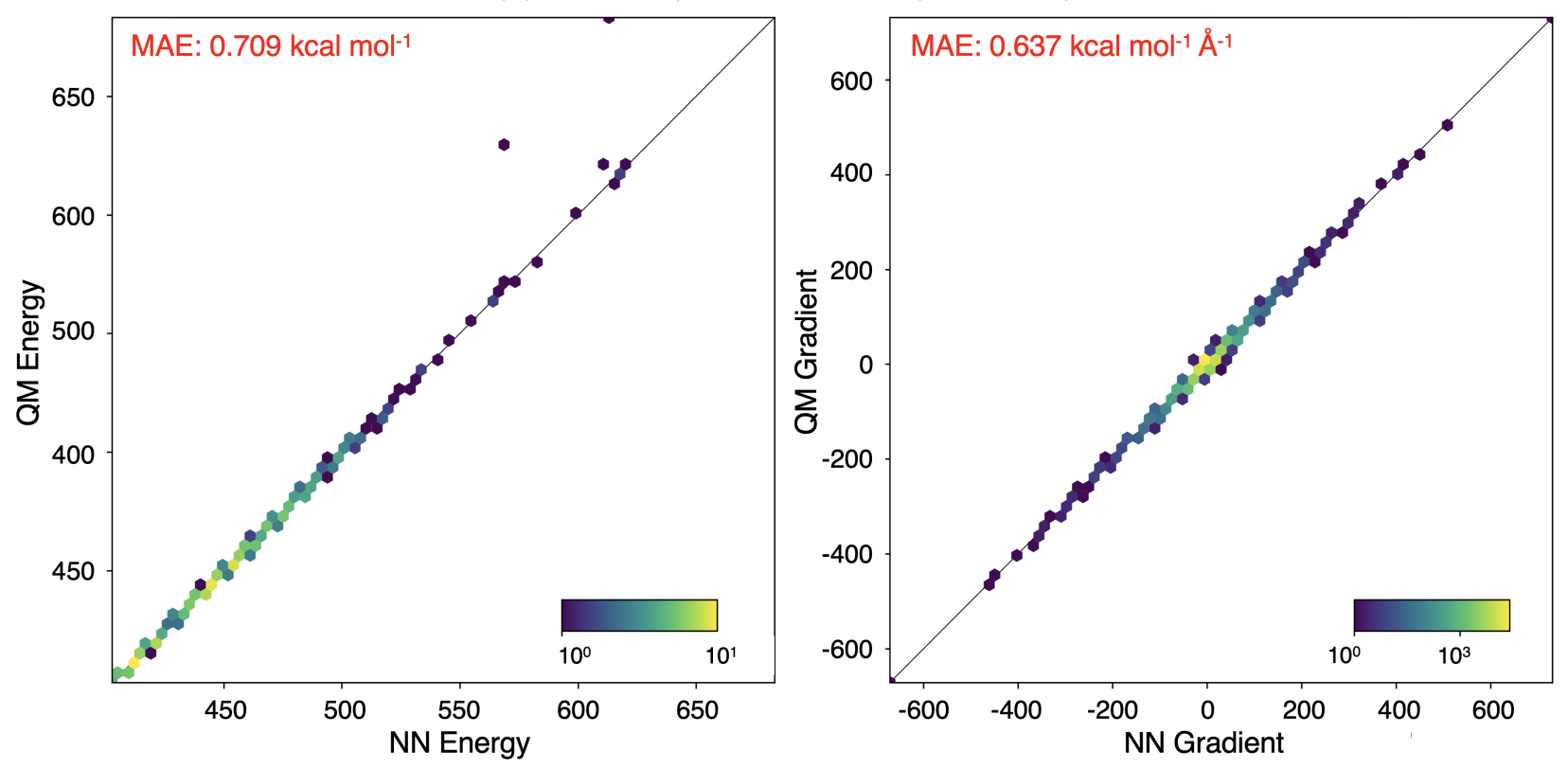

(b) DLPNO-DSD-PBEP86-D3BJ/def2-TZVPD (NN-dh)

Figure 7: Test statistics for transfer learning. Units in $\mathrm{kcal} \mathrm{mol}^{-1}$ and $\mathrm{kcal} \mathrm{mol}^{-1} \AA^{-1}$ for energy and gradient respectively.

Similarly, the transfer-learned NN using datapoints at DLPNO-DSD-PBEP86-D3BJ/def2- 
TZVPD level of theory achieved sub-kcal mol${ }^{-1}$ accuracies for energy and gradients (Figure $7 \mathrm{~b}$ ). This NN will be named NN-dh thereafter, where dh stands for double hybrid.

\section{TS geometries at M06-2X/def2-TZVPD level}

All three TS geometries were refined from the M06-2X/def2-SVP structures using a quantum mechanical EVF algorithm at the M06-2X/def2-TZVPD level of theory. The important bond lengths of the refined geometries were shown in Figure S6. We note that these bond lengths are extremely similar to the corresponding ones optimized at def2-SVP level of theory. The maximum absolute deviation is $0.05 \AA$. Normal mode sampling was carried out with these refined geometries at $298.15 \mathrm{~K}$ before to seed the NRMDs using NN-large/NN-dh.

\section{Trajectories propagated from the vicinity of TS 1 (NN-large and NN-dh)}

Relative to NN-small, the NRMD results of NN-large initiated from the vicinity of TS 1 with Langevin friction coefficient value, $\gamma$, of 0.0012 a.u. show a sharp increase in percentage of recrossed trajectories $(0.11 \%$ v.s. $11.48 \%)$. The mean, standard deviation and median time gaps between C1-C2 and C3-C4 bond formations also increased considerably from 82 to $99 \mathrm{fs}$, 32 to 52 fs and 77 to 86 fs respectively (Figures 6(b) and 9(leftmost plot)). Both the increase of percentages of recrossed trajectories and the increase in bond formation timegaps indicate that the entropic intermediate is further stabilized using a larger basis set, which resulted

in longer lifetimes. As the dicyanoheptafulvene and dimethylfulvene moieties within the entropic intermediate can participate in intramolecular $\pi-\pi$ interactions. These non-covalent interactions are typically described more accurately, and thus stabilized as the basis set size increases. ${ }^{69,70}$

Figure 8 shows the four valid NRMD trajectories initiated with $\gamma$ values of 0.0012 a.u. (corresponding to the friction coefficient of benzene in chloroform) and 0.024 a.u. on NNlarge and NN-dh surfaces. 

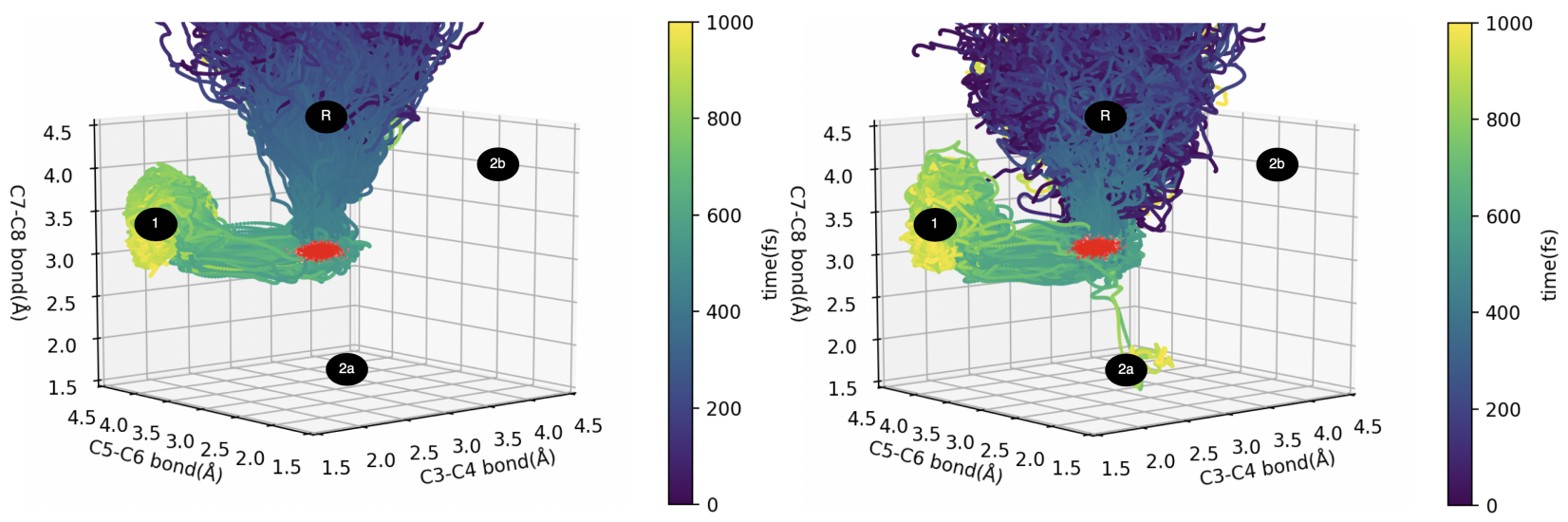

(a) $\gamma=0.0012$ a.u. (M06-2X)

(b) $\gamma=0.024$ a.u. (M06-2X)
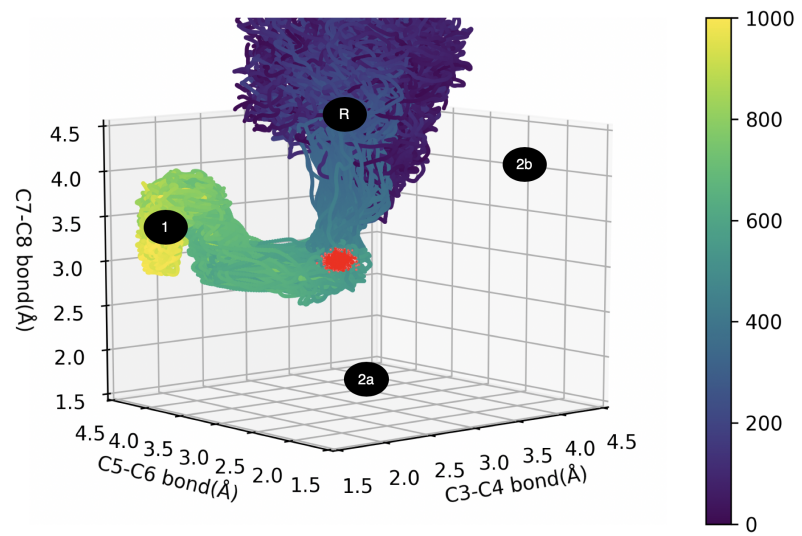

(c) $\gamma=0.0012$ a.u. (DH)

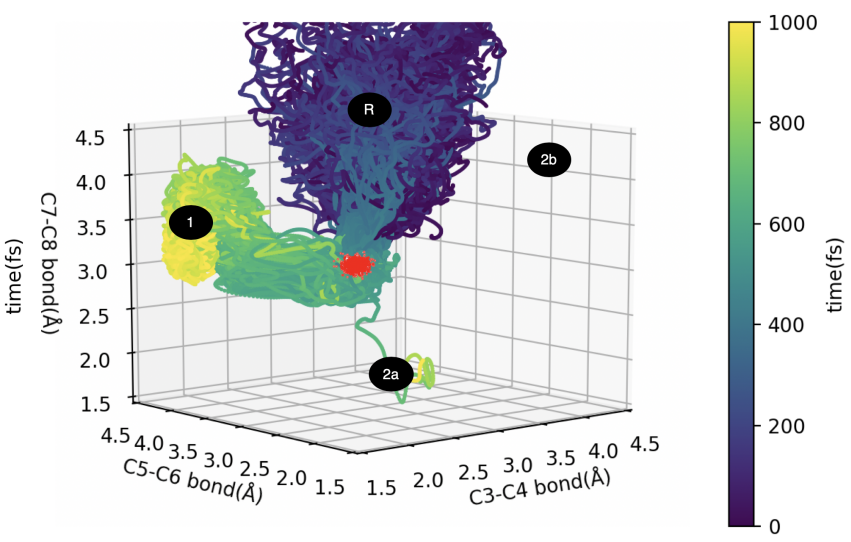

(d) $\gamma=0.024$ a.u. (DH)

Figure 8: Trajectories initiated from the vicinity of TS 1 using NN-large and NN-dh for two Langevin friction coefficients

Bifurcations of the trajectories were not observed when $\gamma=0.0012$ a.u. for either levels of theory. Upon increasing the $\gamma$ values by 20 times, the bifurcation phenomenon began to surface, in which two out of 962 and one out of 752 valid trajectories afforded product $\mathbf{2 a}$ for NN-large and NN-dh respectively. This is due to the increase in lifetime of the entropic intermediate with $\gamma$, which can be quantified by the time gap between $\mathrm{C} 1-\mathrm{C} 2$ and $\mathrm{C} 3-\mathrm{C} 4$ bond formations. For NN-large, the mean and standard deviation (spread in the violin plots) of time gap further increased from 99 to 108 fs and 52 to 64 fs respectively. The median time gap increased from 86 to 91 fs (white dots in the same figure). The mean and median time gaps for NN-dh are extended by 3-4 and 11-13 fs respectively. These are due to the additional dynamical correlation (from the MP2 contribution) and the explicit 
dispersion corrections which are known to increase the magnitude of $\pi-\pi$ interactions, ${ }^{71,72}$ thus prolonging the lifetime of the entropic intermediate. This is also accompanied by a drastic drop in percentage of dynamically concerted trajectories by $16-20 \%$. A detailed summary of time gaps and the percentage of dynamically concerted trajectories is presented in Table S2.

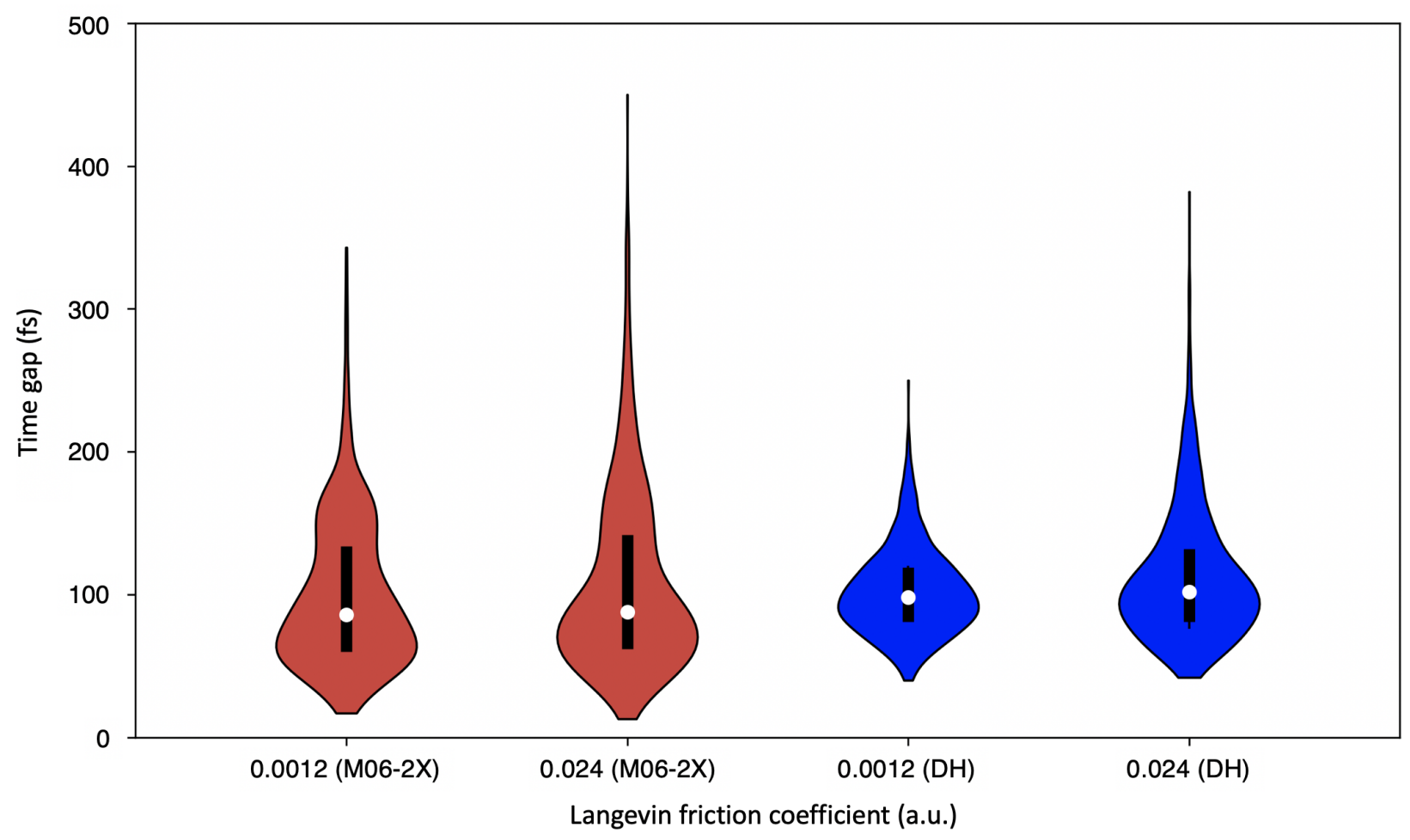

Figure 9: Time gap between C1-C2 and C3-C4 bond formations. M06-2X/def2-TZVPD in red and $\mathrm{DH} /$ def2-TZVPD in blue

Trajectories propagated from the vicinity of TS $2 \mathrm{a}$ and TS $2 \mathrm{~b}$ (NN-large and NN-dh)

Figures S8 and S9 show the trajectories initiated from the vicinity of $\mathbf{T S} \mathbf{2} \mathbf{a}$ and $\mathbf{T S} \mathbf{2 b}$ respectively. Comparing the trajectories of NN-large and the NN-small PESes, there are more trajectories that recrossed back to product 1 from TS $2 \mathbf{2 a}(7.9 \%$ v.s. $3.9 \%)$ and TS 2b (91.1\% v.s. $64.3 \%)$. This can again be explained by the prolonging of the lifetime of the entropic intermediate, which was caused by the larger def2-TZVPD basis set. 
With a 20-time increase in $\gamma$ values, the trajectories become less selective to a particular endpoint. The increase in branching is evident upon comparing a and d subplots in Figures S8 and S9. Similar conclusion were arrived with NN-dh. A full distribution of trajectories is presented in Table S1.

\section{Conclusion}

Dynamical effects on a complex PES with 19 heavy atoms were explored with ML-accelerated DFT simulations. An active learning loop automatically acquired datapoints using a combination of quantum mechanical NEB, neural NEB and NRMD for sampling and iteratively updated a reactive interatomic potential based on GCNN.

Training on 30,973 datapoints, the NN potential reproduced the PES of an ambimodal trispericyclic reaction with $\mathrm{kJ} \mathrm{mol}^{-1}$ accuracy. 3,000 picosecond-long reactive Langevin MD trajectories were used to explore the product distribution. These would have required 3 million DFT simulations in a pure DFT BOMD framework.

Simulations with a friction coefficient corresponding to that of benzene in chloroform, showed no bifurcations in trajectories initiated from TS 1 , TS $2 \mathbf{a}$ and TS $2 \mathbf{b}$. We thus posit that the ambimodal trispericyclic reaction between 8,8-dicyanoheptafulvene and 6,6dimethylfulvene happens in two parallel two-step pathways (Reactants $\longrightarrow$ Product $1 \longrightarrow$ Product 2a, and Reactants $\longrightarrow$ Product $\mathbf{1} \longrightarrow$ Product $\mathbf{2 b}$ ). The higher experimental yield of product $\mathbf{2} \mathbf{a}$ as compared to $\mathbf{2} \mathbf{b}$ can be explained by the larger number of recrossed trajectories initiated from $\mathbf{T S} \mathbf{2} \mathbf{b}$.

Through a transfer learning strategy with datasets of $10 \%$ size, NN potentials of M062x/def2-TZVPD and DLPNO-DSD-PBEP86-D3BJ/def2-TZVPD accuracy were trained (NN-

large and NN-dh). NRMD simulations with NN-large show higher percentage of recrossing trajectories and a longer time gap between bond formations as compared to the original $\mathrm{NN}$-small simulations. NN-dh further increased the time gaps between bond formations. 
These suggest that the stability of the entropic intermediate is related to the strength of intramolecular $\pi-\pi$ interaction between the dicyanoheptafulvene and dimethylfulvene moieties, which is known to increase with basis set size, the inclusion of dispersion correction, and dynamical correlation. By increasing the Langevin friction coefficient, the time gap between bond formations further increased, and some branching to various stationary points was observed.

For NN-large and NN-dh, a total of 12,000 reactive trajectories were generated, which would have required an intractable number of 12 million DFT gradient calls. The combination of sampling with QM and neural-simulators, ${ }^{35,36}$ high-throughput embarrassingly parallel DFT, ${ }^{42}$ active learning, ${ }^{73}$ GCNN potentials ${ }^{28,29}$ and transfer learning ${ }^{26}$ has the potential to speed up ab initio reactive MD by 2 to 3 orders of magnitude, retaining the accuracy of the reference method and to produce novel chemical insight. This opens up avenues for simulating challenging chemical reactions, in which traditional TST fails, with a larger system size and higher level of theory than has been possible so far.

\section{Acknowledgement}

S.J.A. thanks the financial support from the Agency of Science, Technology and Research, Singapore (A*STAR) through the A*STAR Graduate Scholarship (Postdoctoral Fellowship). W.W. thanks the Toyota Research Institute, D. S.-K. thanks the MITEI Seed Fund, S.A. thanks the MIT Buchsbaum Fund and R.G-B thanks MIT DMSE and Toyota Faculty Chair for support. The computations in this paper were executed at the Massachusetts Green High-Performance Computing Center with support from MIT Research Computing.

\section{References}

(1) Baker, J. An algorithm for the location of transition states. Journal of Computational Chemistry 1986, 7, 385-395. 
(2) Berne, B. J.; Ciccotti, G.; Coker, D. F. Classical and Quantum Dynamics in Condensed Phase Simulations; WORLD SCIENTIFIC, 1998.

(3) Behn, A.; Zimmerman, P. M.; Bell, A. T.; Head-Gordon, M. Efficient exploration of reaction paths via a freezing string method. Journal of Chemical Physics 2011, 135.

(4) Mallikarjun Sharada, S.; Zimmerman, P. M.; Bell, A. T.; Head-Gordon, M. Automated transition state searches without evaluating the hessian. Journal of Chemical Theory and Computation 2012, 8, 5166-5174.

(5) Peters, B.; Heyden, A.; Bell, A. T.; Chakraborty, A. A growing string method for determining transition states: Comparison to the nudged elastic band and string methods. Journal of Chemical Physics 2004, 120, 7877-7886.

(6) Hare, S. R.; Tantillo, D. J. Post-transition state bifurcations gain momentum - current state of the field. Pure and Applied Chemistry 2017, 89, 679-698.

(7) Iftimie, R.; Minary, P.; Tuckerman, M. E. Ab initio molecular dynamics: Concepts, recent developments, and future trends. Proceedings of the National Academy of Sciences 2005, 102, 6654-6659.

(8) Marx, D.; Hutter, J. Ab Initio Molecular Dynamics: Basic Theory and Advanced Methods; Cambridge University Press, 2009.

(9) Helgaker, T.; Uggerud, E.; Jensen, H. J. A. Integration of the classical equations of motion on ab initio molecular potential energy surfaces using gradients and Hessians: application to translational energy release upon fragmentation. Chemical Physics Letters 1990, 173, 145-150.

(10) Uggerud, E.; Helgaker, T. Dynamics of the reaction $\mathrm{CH} 2 \mathrm{OH}+\mathrm{CHO}++\mathrm{H} 2$. Translational Energy Release from ab Initio Trajectory Calculations. Journal of the American Chemical Society 1992, 114, 4265-4268. 
(11) Car, R.; Parrinello, M. Unified Approach for Molecular Dynamics and DensityFunctional Theory. Phys. Rev. Lett. 1985, 55, 2471-2474.

(12) Cao, J.; Voth, G. A. The formulation of quantum statistical mechanics based on the Feynman path centroid density. I. Equilibrium properties. The Journal of Chemical Physics 1994, 100, 5093-5105.

(13) Craig, I. R.; Manolopoulos, D. E. Quantum statistics and classical mechanics: Real time correlation functions from ring polymer molecular dynamics. Journal of Chemical Physics 2004, 121, 3368-3373.

(14) Boekelheide, N.; Salomón-Ferrer, R.; Miller, T. F. Dynamics and dissipation in enzyme catalysis. Proceedings of the National Academy of Sciences of the United States of America 2011, 108, 16159-16163.

(15) Hoffmann, R.; Woodward, R. B. Selection Rules for Concerted Cycloaddition Reactions. Journal of the American Chemical Society 1965, 87, 2046-2048.

(16) Hoffmann, H. M. The Ene Reaction. Angewandte Chemie International Edition in English 1969, 8, 556-577.

(17) Gonzalez-James, O. M.; Kwan, E. E.; Singleton, D. A. Entropic intermediates and hidden rate-limiting steps in seemingly concerted cycloadditions. Observation, prediction, and origin of an isotope effect on recrossing. Journal of the American Chemical Society 2012, 134, 1914-1917.

(18) Hare, S. R.; Li, A.; Tantillo, D. J. Post-transition state bifurcations induce dynamical detours in Pummerer-like reactions. Chemical Science 2018, 9, 8937-8945.

(19) Tan, J. S.; Hirvonen, V.; Paton, R. S. Dynamic Intermediates in the Radical Cation Diels-Alder Cycloaddition: Lifetime and Suprafacial Stereoselectivity. Organic Letters 2018, 20, 2821-2825. 
(20) Yang, Z.; Jamieson, C. S.; Xue, X.-S.; Garcia-Borràs, M.; Benton, T.; Dong, X.; Liu, F.; Houk, K. Mechanisms and Dynamics of Reactions Involving Entropic Intermediates. Trends in Chemistry 2019, 1, 22-34.

(21) Abadi, M. et al. TensorFlow: Large-Scale Machine Learning on Heterogeneous Systems. 2015; http://tensorflow.org/, Software available from tensorflow.org.

(22) Paszke, A. et al. In Advances in Neural Information Processing Systems 32; Wallach, H., Larochelle, H., Beygelzimer, A., dAlché-Buc, F., Fox, E., Garnett, R., Eds.; Curran Associates, Inc., 2019; pp 8024-8035.

(23) Behler, J.; Parrinello, M. Generalized neural-network representation of highdimensional potential-energy surfaces. Physical Review Letters 2007, 98, 1-4.

(24) Smith, J. S.; Isayev, O.; Roitberg, A. E. ANI-1: an extensible neural network potential with DFT accuracy at force field computational cost. Chemical Science 2017, 8, 31923203.

(25) Smith, J. S.; Nebgen, B.; Lubbers, N.; Isayev, O.; Roitberg, A. E. Less is more: Sampling chemical space with active learning. The Journal of Chemical Physics 2018, 148, 241733.

(26) Smith, J. S.; Nebgen, B. T.; Zubatyuk, R.; Lubbers, N.; Devereux, C.; Barros, K.; Tretiak, S.; Isayev, O.; Roitberg, A. E. Approaching coupled cluster accuracy with a general-purpose neural network potential through transfer learning. Nature Communications 2019, 10, 1-8.

(27) Devereux1, C.; Smith2, J. S.; Davis, K. K.; Barros3, K.; Zubatyuk4, R.; Isayev4, O.; Roitberg1, A. E. Extending the applicability of the ANI deep learning molecular potential to Sulfur and Halogens. 2020, 
(28) Schütt, K. T.; Arbabzadah, F.; Chmiela, S.; Müller, K. R.; Tkatchenko, A. Quantumchemical insights from deep tensor neural networks. Nature Communications 2017, 8, $6-13$.

(29) Schütt, K. T.; Sauceda, H. E.; Kindermans, P. J.; Tkatchenko, A.; Müller, K. R. SchNet - A deep learning architecture for molecules and materials. Journal of Chemical Physics 2018,148 .

(30) Schütt, K. T.; Kessel, P.; Gastegger, M.; Nicoli, K. A.; Tkatchenko, A.; Müller, K. R. SchNetPack: A Deep Learning Toolbox for Atomistic Systems. Journal of Chemical Theory and Computation 2019, 15, 448-455.

(31) Zhang, L.; Han, J.; Wang, H.; Car, R.; E, W. Deep Potential Molecular Dynamics: A Scalable Model with the Accuracy of Quantum Mechanics. Physical Review Letters 2018, 120, 143001.

(32) Chen, C.; Ye, W.; Zuo, Y.; Zheng, C.; Ong, S. P. Graph Networks as a Universal Machine Learning Framework for Molecules and Crystals. Chemistry of Materials 2019, 31, 3564-3572.

(33) Wang, W.; Gómez-Bombarelli, R. Coarse-graining auto-encoders for molecular dynamics. npj Computational Materials 2019, 5.

(34) Wang, J.; Olsson, S.; Wehmeyer, C.; Pérez, A.; Charron, N. E.; De Fabritiis, G.; Noé, F.; Clementi, C. Machine Learning of Coarse-Grained Molecular Dynamics Force Fields. ACS Central Science 2019, 5, 755-767.

(35) Noé, F.; Tkatchenko, A.; Müller, K.-R.; Clementi, C. Machine learning for molecular simulation. 2019, 1-28.

(36) Jensen, K. F.; Coley, C. W.; Eyke, N. S. Autonomous discovery in the chemical sciences part I: Progress. Angewandte Chemie International Edition $n / a$. 
(37) Yao, K.; Herr, J. E.; Toth, D. W.; McKintyre, R.; Parkhill, J. The TensorMol-0.1 model chemistry: A neural network augmented with long-range physics. Chemical Science 2018, 9, 2261-2269.

(38) Garrido Torres, J. A.; Jennings, P. C.; Hansen, M. H.; Boes, J. R.; Bligaard, T. LowScaling Algorithm for Nudged Elastic Band Calculations Using a Surrogate Machine Learning Model. Phys. Rev. Lett. 2019, 122, 156001.

(39) Koistinen, O. P.; Ásgeirsson, V.; Vehtari, A.; Jónsson, H. Nudged Elastic Band Calculations Accelerated with Gaussian Process Regression Based on Inverse Interatomic Distances. Journal of Chemical Theory and Computation 2019, 15, 6738-6751.

(40) Hachmann, J.; Olivares-Amaya, R.; Atahan-Evrenk, S.; Amador-Bedolla, C.; SánchezCarrera, R. S.; Gold-Parker, A.; Vogt, L.; Brockway, A. M.; Aspuru-Guzik, A. The harvard clean energy project: Large-scale computational screening and design of organic photovoltaics on the world community grid. Journal of Physical Chemistry Letters 2011, 2, 2241-2251.

(41) Lin, K.; Gómez-Bombarelli, R.; Beh, E. S.; Tong, L.; Chen, Q.; Valle, A.; AspuruGuzik, A.; Aziz, M. J.; Gordon, R. G. A redox-flow battery with an alloxazine-based organic electrolyte. Nature Energy 2016, 1, 1-8.

(42) Gómez-Bombarelli, R. et al. Design of efficient molecular organic light-emitting diodes by a high-throughput virtual screening and experimental approach. Nature Materials 2016, 15, 1120-1127.

(43) Pun, G. P.; Batra, R.; Ramprasad, R.; Mishin, Y. Physically informed artificial neural networks for atomistic modeling of materials. Nature Communications 2019, 10, 1-10.

(44) Wei, J. N.; Duvenaud, D. Neural Networks for the Prediction of Organic Chemistry Reactions. 2016, 
(45) Ioannidis, E. I.; Gani, T. Z.; Kulik, H. J. molSimplify: A toolkit for automating discovery in inorganic chemistry. Journal of Computational Chemistry 2016, 2106-2117.

(46) Friederich, P.; dos Passos Gomes, G.; De Bin, R.; Aspuru-Guzik, A.; Balcells, D. Machine Learning Reactivity in the Chemical Space Surrounding Vaska's Complex. 2019; https://chemrxiv.org/articles/Machine_Learning_Reactivity_in_ the_Chemical_Space_Surrounding_Vaska_s_Complex/10347566/1.

(47) Tabor, D. P.; Gómez-Bombarelli, R.; Tong, L.; Gordon, R. G.; Aziz, M. J.; AspuruGuzik, A. Mapping the frontiers of quinone stability in aqueous media: implications for organic aqueous redox flow batteries. Journal of Materials Chemistry A 2019, 7, $12833-12841$.

(48) Grambow, C.; Pattanaik, L.; Green, W. H. Deep Learning of Activation Energies Deep Learning of Activation Energies. 2020,

(49) Liu, C. Y.; Ding, S. T. Cycloadditions of electron-deficient 8,8-disubstituted heptafulvenes to electron-rich 6,6-disubstituted fulvenes. The Journal of Organic Chemistry 1992, 57, 4539-4544.

(50) Xue, X.-S.; Jamieson, C. S.; Garcia-Borràs, M.; Dong, X.; Yang, Z.; Houk, K. N. Ambimodal Trispericyclic Transition State and Dynamic Control of Periselectivity. Journal of the American Chemical Society 2019, 141, 1217-1221.

(51) Duvenaud, D. K.; Maclaurin, D.; Aguilera-Iparraguirre, J.; Gómez-Bombarelli, R.; Hirzel, T.; Aspuru-Guzik, A.; Adams, R. P. Convolutional Networks on Graphs for Learning Molecular Fingerprints. Advances in Neural Information Processing Systems. 2015; pp 2215-2223.

(52) Gilmer, J.; Schoenholz, S. S.; Riley, P. F.; Vinyals, O.; Dahl, G. E. Neural Message Passing for Quantum Chemistry. 34th International Conference on Machine Learning, ICML 2017 2017, 3, 2053-2070. 
(53) Goerigk, L.; Hansen, A.; Bauer, C.; Ehrlich, S.; Najibi, A.; Grimme, S. A look at the density functional theory zoo with the advanced GMTKN55 database for general main group thermochemistry, kinetics and noncovalent interactions. Physical Chemistry Chemical Physics 2017, 19, 32184-32215.

(54) Neese, F. The ORCA program system. Wiley Interdisciplinary Reviews: Computational Molecular Science 2012, 2, 73-78.

(55) Neese, F. Software update: the ORCA program system, version 4.0. Wiley Interdisciplinary Reviews: Computational Molecular Science 2018, 8, e1327.

(56) Zhao, Y.; Truhlar, D. G. The M06 suite of density functionals for main group thermochemistry, thermochemical kinetics, noncovalent interactions, excited states, and transition elements: two new functionals and systematic testing of four M06-class functionals and 12 other function. Theoretical Chemistry Accounts 2008, 120, 215-241.

(57) Wheeler, S. E.; Houk, K. N. Integration grid errors for meta-gga-predicted reaction energies: Origin of grid errors for the M06 suite of functionals. Journal of Chemical Theory and Computation 2010, 6, 395-404.

(58) Mardirossian, N.; Head-Gordon, M. How Accurate Are the Minnesota Density Functionals for Noncovalent Interactions, Isomerization Energies, Thermochemistry, and Barrier Heights Involving Molecules Composed of Main-Group Elements? Journal of Chemical Theory and Computation 2016, 12, 4303-4325.

(59) Smidstrup, S.; Pedersen, A.; Stokbro, K.; Jónsson, H. Improved initial guess for minimum energy path calculations. The Journal of Chemical Physics 2014, 140, 214106.

(60) Landrum, G. RDKit: Open-source cheminformatics. http://www.rdkit.org.

(61) Halgren, T. A. Merck molecular force field. I. Basis, form, scope, parameterization, and performance of MMFF94. Journal of Computational Chemistry 1996, 17, 490-519. 
(62) Kingma, D. P.; Ba, J. L. Adam: A method for stochastic optimization. 3rd International Conference on Learning Representations, ICLR 2015 - Conference Track Proceedings 2015, 1-15.

(63) Larsen, A. H. et al. The atomic simulation environment - a Python library for working with atoms. Journal of Physics: Condensed Matter 2017, 29, 273002.

(64) Henkelman, G.; Jónsson, H. Improved tangent estimate in the nudged elastic band method for finding minimum energy paths and saddle points. The Journal of Chemical Physics 2000, 113, 9978-9985.

(65) Hase, W. L.; Duchovic, R. J.; Hu, X.; Komornicki, A.; Lim, K. F.; h Lu, D.; Peslherbe, G. H.; Swamy, K. S. N.; Linde, S. R. V.; Varandas, A. J. C.; Wang, H.; Wolf, R. J. VENUS96: A General Chemical Dynamics Computer Program. 1996.

(66) Safi, A.; Nicolas, C.; Neau, E.; Chevalier, J.-l. Diffusion Coefficients of Organic Compounds at Infinite Dilution in Mixtures Involving Associating Compounds. Experimental Determination and Modeling by Group Contribution Methods. Journal of Chemical E) Engineering Data 2008, 53, 444-448.

(67) Ribeiro, R. F.; Marenich, A. V.; Cramer, C. J.; Truhlar, D. G. Use of Solution-Phase Vibrational Frequencies in Continuum Models for the Free Energy of Solvation. The Journal of Physical Chemistry B 2011, 115, 14556-14562.

(68) Grimme, S. Supramolecular Binding Thermodynamics by Dispersion-Corrected Density Functional Theory. Chemistry $\hat{a}$ A European Journal 2012, 18, 9955-9964.

(69) Sinnokrot, M. O.; Valeev, E. F.; Sherrill, C. D. Estimates of the ab initio limit for $\pi-\pi$ interactions: The benzene dimer. Journal of the American Chemical Society 2002, 124, $10887-10893$. 
(70) Sherrill, C. D. Reviews in Computational Chemistry; John Wiley Sons, Ltd, 2009; Chapter 1, pp 1-38.

(71) Ye, X.; Li, Z. H.; Wang, W.; Fan, K.; Xu, W.; Hua, Z. The parallel $\pi-\pi$ stacking: A model study with MP2 and DFT methods. Chemical Physics Letters 2004, 397, 56-61.

(72) Wang, W.; Zhang, Y.; Wang, Y. B. The $\pi \cdots \pi$ Stacking interactions between homogeneous dimers of $\operatorname{C6FxI}(6-\mathrm{x})(\mathrm{x}=0,1,2,3,4$, and 5$)$ : A comparative study with the halogen bond. Journal of Physical Chemistry A 2012, 116, 12486-12491.

(73) Häse, F.; Roch, L. M.; Kreisbeck, C.; Aspuru-Guzik, A. Phoenics: A Bayesian Optimizer for Chemistry. ACS Central Science 2018, 4, 1134-1145. 